Analele Universităţii de Vest, Timişoara

Seria Matematică - Informatică LIV , 1, (2016), 3- 16

\title{
On the Local Convergence of an Eighth-order Method for Solving Nonlinear Equations
}

\author{
Ioannis K. Argyros, Munish Kansal, and V. Kanwar
}

\begin{abstract}
We present a local convergence analysis of an eighthorder method for approximating a locally unique solution of a nonlinear equation. Earlier studies such as have shown convergence of these methods under hypotheses up to the seventh derivative of the function although only the first derivative appears in the method. In this study, we expand the applicability of these methods using only hypotheses up to the first derivative of the function. This way the applicability of these methods is extended under weaker hypotheses. Moreover, the radius of convergence and computable error bounds on the distances involved are also given in this study. Numerical examples are also presented in this study.
\end{abstract}

AMS Subject Classification (2000). 65D10; 65D99

Keywords. Nonlinear equation, Optimal efficiency index, Local convergence, Eighth order of convergence

\section{Introduction}

In this study, we are concerned with the problem of approximating a locally unique solution $\xi$ of equation

$$
f(x)=0,
$$

where $f: D \subseteq S \rightarrow S$ is a differentiable nonlinear function and $D$ is a convex subset of $S$ and $S$ is $\mathbb{R}$ or $\mathbb{C}$. Newton-like methods are famous for finding the 
solution of (1.1). The study about convergence matter of iterative procedures is usually based on two types: semi-local and local convergence analysis. The semi-local convergence matter is, based on the information around an initial point, to give conditions ensuring the convergence of the iterative procedure; while the local one is, based on the information around a solution, to find estimates of the radii of convergence balls. There exist many studies which deal with the local and semi-local convergence analysis of Newton-like methods such as [1-24].

Third-order methods such as Euler's, Ostrowski's square root, Laguerre's method [16] require the computation of second-derivative $f^{\prime \prime}$ at each step, which in general is very expensive. That is why many authors have developed and analyzed higher order multipoint methods [20]. In this paper, we introduce the method defined for each $n=0,1,2, \ldots$ by

$$
\left\{\begin{array}{l}
y_{n}=x_{n}-\frac{f\left(x_{n}\right)}{f^{\prime}\left(x_{n}\right)}, \\
t_{n}=y_{n}-\frac{f\left(y_{n}\right)\left(f\left(x_{n}\right)+\beta f\left(y_{n}\right)\right)}{f^{\prime}\left(x_{n}\right)\left(f\left(x_{n}\right)+(\beta-2) f\left(y_{n}\right)\right)} \\
x_{n+1}=t_{n}-\delta \frac{H_{3}\left(t_{n}\right)}{f^{\prime}\left(t_{n}\right)},
\end{array}\right.
$$

where $x_{0}$ is an initial point, $\beta, \delta \in S$ are parameters. Here, $f[x, y]$ denotes a divided difference of order one for $f$ at the points $x, y$ and

$$
\begin{aligned}
H_{3}\left(t_{n}\right) & =f\left(x_{n}\right)+f^{\prime}\left(x_{n}\right) \frac{\left(t_{n}-y_{n}\right)^{2}\left(t_{n}-x_{n}\right)}{\left(y_{n}-x_{n}\right)\left(x_{n}+2 y_{n}-3 t_{n}\right)}+f^{\prime}\left(t_{n}\right) \frac{\left(t_{n}-y_{n}\right)\left(x_{n}-t_{n}\right)}{x_{n}+2 y_{n}-3 t_{n}} \\
& -f\left[x_{n}, y_{n}\right] \frac{\left(t_{n}-y_{n}\right)^{3}}{\left(y_{n}-x_{n}\right)\left(x_{n}+2 y_{n}-3 t_{n}\right)} .
\end{aligned}
$$

The first two steps of method (1.2) are same as that of King's family. Notice that King's class of methods can be obtained from method (1.2), if $\delta=0$. Moreover, if $\delta=1$, we obtain methods proposed in [18] and [19].

From a computational point of view, the method (1.2) attains eighthorder of convergence using only four functional evaluations, viz. $f\left(x_{n}\right)$, $f^{\prime}\left(x_{n}\right), \quad f\left(y_{n}\right)$ and $f\left(t_{n}\right)$, per iteration. Therefore, optimal efficiency index [24] of the proposed class is $E=\sqrt[4]{8} \approx 1.682$. Earlier studies showed the eighth-order of convergence of method (1.2) using Taylor expansions and hypotheses reaching up to the seventh derivative of the function $f$. These hypotheses limit the applicability of method (1.2).

As a motivational example, define function $f$ on $X=\left[-\frac{1}{2}, \frac{5}{2}\right]$ by

$$
f(x)= \begin{cases}x^{3} \ln x^{2}+x^{5}-x^{4}, & x \neq 0 \\ 0, & x=0\end{cases}
$$


Let $\xi=1$. We have that

$$
\begin{aligned}
& f^{\prime}(x)=3 x^{2} \ln x^{2}+5 x^{4}-4 x^{3}+2 x^{2}, \\
& f^{\prime \prime}(x)=6 x \ln x^{2}+20 x^{3}-12 x^{2}+10 x, \\
& f^{\prime \prime \prime}(x)=6 \ln x^{2}+60 x^{2}-24 x+22 .
\end{aligned}
$$

Then, obviously function $f$ does not have bounded third derivative in $X$. Notice that, in particular there is a plethora of iterative methods for approximating solutions of nonlinear equations on $X$. These results show that if initial point $x_{0}$ is sufficiently close to the solution $\xi$, then the sequence $\left\{x_{n}\right\}$ converges to $\xi$. But how close to the solution $\xi$, the initial guess $x_{0}$ should be? These local results give no information on the radius of convergence ball for the corresponding method. We address this question for method (1.2) in Section 2. The same technique can be used to other methods. In the present study, we extend the applicability of these methods by using hypotheses up to the first derivative of function $f$ and contractions. Moreover, we avoid Taylor expansions and use instead Lipschitz parameters. This way we do not have to use higher order derivatives to show the convergence of these methods.

The rest of the paper is organized as follows: In Section 2, we present the local convergence of method (1.2). The numerical examples are presented in the concluding Section 3.

\section{Local convergence analysis}

We present the local convergence analysis of method (1.2) in this section.

Let $U(v, \rho)$ and $\bar{U}(v, \rho)$ denote the open and closed balls in $S$, respectively, with center $v \in S$ and radius $\rho>0$. Let $L_{0}>0, L>0, M_{0}>0, \gamma>$ $0, \beta, \delta \in S$ and $\alpha \in\left(0, \frac{1}{3}\right)$ be parameters. It is convenient for the local convergence analysis of method (1.2) to define some functions and parameters. Define functions on the interval $\left[0, \frac{1}{L_{0}}\right)$ by

$$
\begin{aligned}
g_{1}(t) & =\frac{L t}{2\left(1-L_{0} t\right)}, \\
q(t) & =\frac{L_{0}}{2} t+M|\beta-2| g_{1}(t), \\
q_{1}(t) & =q(t)-1,
\end{aligned}
$$

and parameter $r_{1}$ by

$$
r_{1}=\frac{2}{2 L_{0}+L}<\frac{1}{L_{0}} .
$$


Then, we have that $g_{1}\left(r_{1}\right)=1$ and $0 \leq g_{1}(t)<1$ for each $t \in\left[0, r_{1}\right)$. We also get that $q_{1}(0)=-1<0$ and $q_{1}(t) \rightarrow \infty$ as $t \rightarrow \frac{1^{-}}{L_{0}}$. It follows from the intermediate value theorem that function $q_{1}(t)$ has zeros in the interval $\left(0, \frac{1}{L_{0}}\right)$. Denote by $r_{q}$ the smallest such zero. Then, we have that $q_{1}\left(r_{q}\right)=1$ and $0 \leq q(t)<1$ for each $t \in\left[0, r_{q}\right)$.

Case 1: $r_{1} \leq r_{q}$.

Then, define functions on the interval $\left[0, r_{1}\right)$ by

$$
g_{2}(t)=\left[1+\frac{M^{2}\left(1+|\beta| g_{1}(t)\right)}{\left(1-L_{0} t\right)(1-q t)}\right]
$$

and $h_{2}(t)=g_{2}(t)-1$.

Then, we get that $h_{2}(0)=-1<0$ and $h_{2}(t) \rightarrow \infty$ as $t \rightarrow r_{1}^{-}$. Hence, function $h_{2}$ has a smallest zero $r_{2} \in\left(0, r_{1}\right)$.

Case 2: $r_{q}<r_{1}$.

Similarly, $h_{2}(0)=-1<0$ and $h_{2}(t) \rightarrow \infty$ as $t \rightarrow r_{q}^{-}$. Hence, function $h_{2}$ has a smallest zero $r_{2} \in\left(0, r_{q}\right)$. Finally, in either case define functions

$$
\begin{aligned}
g_{3}(t) & =g_{2}(t)+M|\delta|\left[\frac{1}{1-L_{0} t}+\frac{\alpha \gamma M^{3} g_{1}(t)^{2}\left(1+|\beta| g_{1}(t)\right)}{\left(1-\frac{L_{0} t}{2}\right)(1-q(t))^{2}}\right. \\
& \left.+\frac{\alpha \gamma M^{2} g_{1}(t)\left(1+|\beta| g_{1}(t)\right)}{\left(1-L_{0} t\right)(1-q(t))}+\frac{\alpha M_{0} M^{3}\left(1+|\beta-1| g_{1}(t)+|\beta| g_{1}(t)^{2}\right)^{2}}{\left(1-L_{0} t\right)\left(1-\frac{L_{0} t}{2}\right)(1-q(t))^{2}}\right]
\end{aligned}
$$

and $h_{3}(t)=g_{3}(t)-1$.

Suppose that

$$
M|\delta|\left(1+M_{0} M^{3}\right)<1 .
$$

Then, $h_{3}(0)=M|\delta|\left(1+M_{0} M^{3}\right)-1<0$ and $h_{3}(t) \rightarrow+\infty$ as $t \rightarrow r_{1}^{-}$(under case 1 ) or as $t \rightarrow r_{q}^{-}$(under case 2 ). Denote by $r_{3}$ the smallest such zero. Set

$$
r=\min \left\{r_{1}, r_{2}, r_{3}, r_{q}\right\}
$$

Then, we have that

$$
\begin{aligned}
& 0 \leq g_{1}(t)<1 \\
& 0 \leq q(t)<1 \\
& 0 \leq g_{2}(t)<1
\end{aligned}
$$

and

$$
0 \leq g_{3}(t), \text { for each } t \in[0, r)
$$


It is convenient to rewrite method (1.2) as

$$
\begin{aligned}
& y_{n}=x_{n}-\frac{f\left(x_{n}\right)}{f^{\prime}\left(x_{n}\right)}, \\
& t_{n}=y_{n}-\frac{f\left(y_{n}\right)\left(f\left(x_{n}\right)+\beta f\left(y_{n}\right)\right)}{f^{\prime}\left(x_{n}\right)\left(f\left(x_{n}\right)+(\beta-2) f\left(y_{n}\right)\right)}, \\
& x_{n+1}=t_{n}-\delta\left(A_{n}+B_{n}+C_{n}+D_{n}\right),
\end{aligned}
$$

where

$$
\left\{\begin{aligned}
A_{n} & =-\frac{f\left(x_{n}\right)}{f^{\prime}\left(x_{n}\right)} \\
B_{n} & =-\frac{\left(t_{n}-x_{n}\right)^{2}\left(t_{n}-x_{n}\right)}{\left(y_{n}-x_{n}\right)\left(x_{n}+2 y_{n}-3 t_{n}\right)} \\
C_{n} & =-\frac{f\left(t_{n}\right)\left(t_{n}-y_{n}\right)\left(x_{n}-t_{n}\right)}{f^{\prime}\left(x_{n}\right)\left(x_{n}+2 y_{n}-3 t_{n}\right)} \\
D_{n} & =\frac{f\left[x_{n}, y_{n}\right]\left(t_{n}-y_{n}\right)^{3}}{f^{\prime}\left(x_{n}\right)\left(y_{n}-x_{n}\right)\left(x_{n}+2 y_{n}-3 t_{n}\right)}
\end{aligned}\right.
$$

In view of the definitions of $x_{n}, y_{n}, t_{n}, B_{n}, C_{n}, D_{n}$ and by simple algebraic manipulations, we can also write that

$$
\begin{aligned}
B_{n} & =\frac{f\left(y_{n}\right)^{2}\left(f\left(x_{n}\right)+\beta f\left(y_{n}\right)\right)^{2}\left(f\left(x_{n}\right)^{2}+(\beta-1) f\left(x_{n}\right) f\left(y_{n}\right)+\beta f\left(y_{n}\right)^{2}\right)}{f\left(x_{n}\right)\left(f\left(x_{n}\right)+(\beta-2) f\left(y_{n}\right)\right)^{2}\left(f\left(x_{n}\right)^{2}+(\beta+1) f\left(x_{n}\right) f\left(y_{n}\right)+3 \beta f\left(y_{n}\right)^{2}\right)}, \\
C_{n} & =\frac{f^{\prime}\left(t_{n}\right) f\left(y_{n}\right)\left(f\left(x_{n}\right)+\beta f\left(y_{n}\right)\right)\left(f\left(x_{n}\right)^{2}+(\beta-1) f\left(x_{n}\right) f\left(y_{n}\right)+\beta f\left(y_{n}\right)^{2}\right)}{f^{\prime}\left(x_{n}\right)\left(f\left(x_{n}\right)+(\beta-2) f\left(y_{n}\right)\right)^{2}\left(f\left(x_{n}\right)^{2}+(\beta+1) f\left(x_{n}\right) f\left(y_{n}\right)+3 \beta f\left(y_{n}\right)^{2}\right)}
\end{aligned}
$$

and

$$
D_{n}=\frac{f\left[x_{n} ; y_{n}\right]\left(f\left(x_{n}\right)^{2}+(\beta-1) f\left(x_{n}\right) f\left(y_{n}\right)+\beta f\left(y_{n}\right)^{2}\right)^{3}}{f\left(x_{n}\right) f^{\prime}\left(x_{n}\right)^{2}\left(f\left(x_{n}\right)+(\beta-2) f\left(y_{n}\right)\right)^{2}\left(f\left(x_{n}\right)^{2}+(\beta+1) f\left(x_{n}\right) f\left(y_{n}\right)+3 \beta f\left(y_{n}\right)^{2}\right)} .
$$

Next, we shall present the local convergence analysis of method (1.2) using previous notations.

Theorem 2.1. Let $f: D \subseteq S \rightarrow S$ be a differentiable function. Suppose that there exists a divided difference of order one $f[\cdot ; \cdot]: D \times D \rightarrow L(D), \xi \in D$, $L_{0}>0, L>0, M_{0}>0, M>0, \gamma>0, \beta, \delta \in S$ and $\alpha \in\left(0, \frac{1}{3}\right)$ such that

$$
\begin{gathered}
M|\delta|\left(1+\alpha M_{0} M^{3}\right)<1, \\
\max \left\{\beta_{0}, 5-2 \sqrt{6}\right\} \leq|\beta| \leq 3+2 \sqrt{2} \\
f(\xi)=0, \quad f^{\prime}(\xi) \neq 0,\left|f^{\prime}(\xi)^{-1}\right| \leq \gamma
\end{gathered}
$$




$$
\begin{gathered}
\left|f^{\prime}(\xi)^{-1}\left(f^{\prime}(x)-f^{\prime}(\xi)\right)\right| \leq L_{0}|x-\xi|, \quad \text { for each } x \in D, \\
\left|f^{\prime}(\xi)^{-1}\left(f^{\prime}(x)-f^{\prime}(y)\right)\right| \leq L|x-y|, \quad \text { for each } x, y \in D_{0}:=D \cap U\left(\xi, \frac{1}{L_{0}}\right), \\
\left|f^{\prime}(\xi)^{-1} f^{\prime}(x)\right| \leq M, \quad \text { for each } x \in D_{0}, \\
\left|f^{\prime}(\xi)^{-1} f[x ; y]\right| \leq M_{0} \quad \text { for each } x, y \in D_{0}
\end{gathered}
$$

and

$$
\bar{U}(\xi, r) \subseteq D,
$$

where the convergence radius $r$ is defined in (2.1) and

$$
\beta_{0}=\frac{(1+\alpha)^{2}}{\sqrt{((1-\alpha)(1-6 \alpha))^{2}-\alpha(\alpha-2)(1+\alpha)^{2}}-(1-\alpha)(1-6 \alpha)} .
$$

Then, the sequence $\left\{x_{n}\right\}$ generated by method (1.2) for $x_{0} \in U(\xi, r)-\{\xi\}$ is well defined, remains in $U(\xi, r)$ for each $n=0,1,2, \ldots$ and converges to $\xi$. Moreover, the following estimates hold

$$
\begin{gathered}
\left|y_{n}-\xi\right| \leq g_{1}\left(\left|x_{n}-\xi\right|\right)\left|x_{n}-\xi\right| \leq\left|x_{n}-\xi\right|<r \\
\left|t_{n}-\xi\right| \leq g_{2}\left(\left|x_{n}-\xi\right|\right)\left|x_{n}-\xi\right| \leq\left|x_{n}-\xi\right|
\end{gathered}
$$

and

$$
\left|x_{n+1}-\xi\right| \leq g_{3}\left(\left|x_{n}-\xi\right|\right)\left|x_{n}-\xi\right| \leq\left|x_{n}-\xi\right|,
$$

where the " $g$ " functions are defined previously. Furthermore, for $T \in\left[r, \frac{2}{L_{0}}\right)$, $\xi$ is the only solution of equation $f(x)=0$ in $D_{1}:=\bar{U}(\xi, T) \cap D$.

Proof. We shall show estimates (2.19)-(2.21) using mathematical induction. By hypothesis $x_{0} \in U(\xi, r)-\{\xi\}$, the definition of $r$ and (2.13), we get that

$$
\left|f^{\prime}(\xi)^{-1}\left(f^{\prime}\left(x_{0}\right)-f^{\prime}(\xi)\right)\right| \leq L_{0}\left|x_{0}-\xi\right|<L_{0} r<1 .
$$

It follows from (2.22) and the Banach lemma on invertible functions [7],[10], [21], [22], [24] that $f^{\prime}\left(x_{0}\right) \neq 0$ and

$$
\left|f^{\prime}\left(x_{0}\right)^{-1} f^{\prime}(\xi)\right| \leq \frac{1}{1-L_{0}\left|x_{0}-\xi\right|} .
$$

Hence, $y_{0}$ is well defined by the first sub-step of method (1.2) for $n=0$. We can write

$$
y_{0}-\xi=x_{0}-\xi-f^{\prime}\left(x_{0}\right)^{-1} f\left(x_{0}\right),
$$


Using (2.2),(2.14),(2.23) and (2.24), we get that

$$
\begin{aligned}
\left|y_{0}-\xi\right| & \leq\left|x_{0}-\xi-f^{\prime}\left(x_{0}\right)^{-1} f^{\prime}(\xi)\right|, \\
& \left.\leq\left|f^{\prime}\left(x_{0}\right)^{-1} f^{\prime}(\xi)\right| \mid \int_{0}^{1} f^{\prime}(\xi)^{-1}\left[f^{\prime}\left(\xi+\theta\left(x_{0}-\xi\right)\right)-f^{\prime}\left(x_{0}\right)\right)\right]\left(x_{0}-\xi\right) d \theta \mid, \\
& \leq \frac{L\left|x_{0}-\xi\right|^{2}}{2\left(1-L_{0}\left|x_{0}-\xi\right|\right)} \\
& =g_{1}\left(\left|x_{0}-\xi\right|\right)\left|x_{0}-\xi\right|<\left|x_{0}-\xi\right|<r,
\end{aligned}
$$

which shows (2.19) for $n=0$ and $y_{0} \in U(\xi, r)$. Using (2.12), we can write that

$$
f\left(x_{0}\right)=f\left(x_{0}\right)-f(\xi)=\int_{0}^{1} f^{\prime}\left(\xi+\theta\left(x_{0}-\xi\right)\right)\left(x_{0}-\xi\right) d \theta
$$

In view of (2.15) and (2.26), we get that

$$
\begin{aligned}
\left|f^{\prime}(\xi)^{-1} f\left(x_{0}\right)\right| & \leq\left|\int_{0}^{1} f^{\prime}(\xi)^{-1} f\left(\xi+\theta\left(x_{0}-\xi\right)\right)\left(x_{0}-\xi\right) d \theta\right| \\
& \leq M\left|x_{0}-\xi\right|
\end{aligned}
$$

and similarly

$$
\left|f^{\prime}(\xi)^{-1} f\left(y_{0}\right)\right| \leq M\left|y_{0}-\xi\right|
$$

Then, by (2.16), we have that

$$
\left|f^{\prime}(\xi)^{-1} f\left[x_{0}, y_{0}\right]\right| \leq M_{0}\left|y_{0}-\xi\right|
$$

Next, we shall show that $f\left(x_{0}\right)+(\beta-2) f\left(y_{0}\right)$ is invertible. Using (2.3), (2.12), (2.13), (2.25) and (2.28), we get that

$$
\begin{aligned}
& \left|\left(f^{\prime}(\xi)\left(x_{0}-\xi\right)\right)^{-1}\left[f\left(x_{0}\right)-f(\xi)-f^{\prime}(\xi)\left(x_{0}-\xi\right)+(\beta-2) f\left(y_{0}\right)\right]\right| \\
& \leq\left|x_{0}-\xi\right|^{-1}\left(\frac{L_{0}}{2}\left|x_{0}-\xi\right|^{2}+|\beta-2| M\left|y_{0}-\xi\right|\right) \\
& \leq\left|x_{0}-\xi\right|^{-1}\left(\frac{L_{0}}{2}\left|x_{0}-\xi\right|^{2}+|\beta-2| M g_{1}\left(\left|x_{0}-\xi\right|\right)\left|x_{0}-\xi\right|\right) \\
& =q\left(\left|x_{0}-\xi\right|\right)<1 .
\end{aligned}
$$

It follows from (2.30) that $f\left(x_{0}\right)+(\beta-2) f\left(y_{0}\right)$ is invertible and

$$
\left|\left(f\left(x_{0}\right)+(\beta-2) f\left(y_{0}\right)\right)^{-1} f^{\prime}(\xi)\right| \leq \frac{1}{\left|x_{0}-\xi\right|\left(1-q\left(\left|x_{0}-\xi\right|\right)\right)} .
$$


It follows that $t_{0}$ is well defined from the second substep of method (1.2) for $n=0$. Then, using $(2.4),(2.23),(2.27),(2.28)$ and $(2.31)$, we have that

$$
\begin{aligned}
\left|t_{0}-\xi\right| & \leq\left|y_{0}-\xi\right|+\left|\frac{f^{\prime}(\xi)^{-1} f\left(y_{0}\right) f^{\prime}(\xi)^{-1}\left(f\left(x_{0}\right)+\beta f\left(y_{0}\right)\right)}{f^{\prime}(\xi)^{-1} f\left(x_{0}\right) f^{\prime}(\xi)^{-1}\left(f\left(x_{0}\right)+(\beta-2) f\left(y_{0}\right)\right)}\right| \\
& \leq\left|y_{0}-\xi\right|+\frac{M^{2}\left|y_{0}-\xi\right|\left(\left|x_{0}-\xi\right|+|\beta|\left|y_{0}-\xi\right|\right)}{\left(1-L_{0}\left|x_{0}-\xi\right|\right)\left|x_{0}-\xi\right|\left(1-q\left(\left|x_{0}-\xi\right|\right)\right)} \\
& {\left[1+\frac{M^{2}\left(\left|x_{0}-\xi\right|+|\beta| g_{1}\left(\left|x_{0}-\xi\right|\right)\right)\left|x_{0}-\xi\right|}{\left(1-L_{0}\left|x_{0}-\xi\right|\right)\left|x_{0}-\xi\right|\left(1-q\left(\left|x_{0}-\xi\right|\right)\right)}\right]\left|y_{0}-\xi\right| } \\
& =g_{2}\left(\left|x_{0}-\xi\right|\right)\left|x_{0}-\xi\right|<\left|x_{0}-\xi\right|<r,
\end{aligned}
$$

which shows (2.20) for $n=0$ and $t_{0} \in U(\xi, r)$.

Next we need estimates on $\left|A_{0}\right|,\left|B_{0}\right|,\left|C_{0}\right|$ and $\left|D_{0}\right|$. Suppose $f\left(x_{0}\right) \neq$ 0 . Consider the expressions $f^{2}\left(x_{0}\right)+(\beta+1) f\left(x_{0}\right) f\left(y_{0}\right)+3 \beta f^{2}\left(y_{0}\right)$ and $f^{2}\left(x_{0}\right)+$ $(\beta-1) f\left(x_{0}\right) f\left(y_{0}\right)+\beta f^{2}\left(y_{0}\right)$ as quadratic polynomials in $f\left(y_{0}\right)$ (or $f\left(x_{0}\right)$ ). Then, their discriminants are given, respectively by $\left(\beta^{2}-10 \beta+1\right) f^{2}\left(x_{0}\right)$ and $\left(\beta^{2}-6 \beta+1\right) f^{2}\left(x_{0}\right)$, which are negative by $(2.11)$. Hence,

$$
f^{2}\left(x_{0}\right)+(\beta+1) f\left(x_{0}\right) f\left(y_{0}\right)+3 \beta f^{2}\left(y_{0}\right)>0
$$

and

$$
f^{2}\left(x_{0}\right)+(\beta-1) f\left(x_{0}\right) f\left(y_{0}\right)+\beta f^{2}\left(y_{0}\right)>0
$$

That is $x_{1}$ is well-defined. We also have by (2.33) and (2.34) that

$$
\begin{aligned}
& \left|\frac{f^{2}\left(x_{0}\right)+(\beta-1) f\left(x_{0}\right) f\left(y_{0}\right)+\beta f^{2}\left(y_{0}\right)}{f^{2}\left(x_{0}\right)+(\beta+1) f\left(x_{0}\right) f\left(y_{0}\right)+3 \beta f^{2}\left(y_{0}\right)}\right| \\
& =\frac{f^{2}\left(x_{0}\right)+(\beta-1) f\left(x_{0}\right) f\left(y_{0}\right)+\beta f^{2}\left(y_{0}\right)}{f^{2}\left(x_{0}\right)+(\beta+1) f\left(x_{0}\right) f\left(y_{0}\right)+3 \beta f^{2}\left(y_{0}\right)} \leq \alpha,
\end{aligned}
$$

since (2.35) reduces to showing that

$$
\alpha(\alpha-2) \beta^{2}+2(1-\alpha)(1-6 \alpha) \beta+(1+\alpha)^{2} \leq 0,
$$

which is true for $\beta \geq \beta_{0}\left((2.11)\right.$ and (2.18)). Hence, $x_{1}$ is well-defined by the third substep of method (1.2) for $n=0$. Then, using (2.6), (2.23) and (2.27), we have that

$$
\left|A_{0}\right| \leq \frac{M\left|x_{0}-\xi\right|}{1-L_{0}\left|x_{0}-\xi\right|}
$$

By (2.7), (2.12), (2.23), (2.25), (2.28), (2.31) and (2.35), we have that 


$$
\begin{aligned}
\left|B_{0}\right| & \leq \frac{\alpha \gamma M^{4}\left|y_{0}-\xi\right|\left(\left|x_{0}-\xi\right|+\beta\left|y_{0}-\xi\right|\right)^{2}}{\left|x_{0}-\xi\right|\left(1-\frac{L_{0}\left|x_{0}-\xi\right|}{2}\right)\left|x_{0}-\xi\right|^{2}\left(1-q\left(\left|x_{0}-\xi\right|\right)\right)^{2}} \\
& \leq \frac{\alpha \gamma M^{4} g_{1}^{2}\left(\left|x_{0}-\xi\right|\right)\left(1+|\beta| g_{1}\left(\left|x_{0}-\xi\right|\right)\right)\left|x_{0}-\xi\right|}{\left(1-\frac{L_{0}\left|x_{0}-\xi\right|}{2}\right)\left(1-q\left(\left|x_{0}-\xi\right|\right)\right)} .
\end{aligned}
$$

In view of $(2.8),(2.12),(2.23),(2.25),(2.28),(2.31)$ and $(2.35)$, we obtain that

$$
\begin{aligned}
\left|C_{0}\right| & \leq \frac{\alpha \gamma M^{3}\left|y_{0}-\xi\right|\left(\left|x_{0}-\xi\right|+\beta\left|y_{0}-\xi\right|\right)}{\left(1-L_{0}\left|x_{0}-\xi\right|\right)\left|x_{0}-\xi\right|\left(1-q\left(\left|x_{0}-\xi\right|\right)\right)} \\
& \leq \frac{\alpha \gamma M^{3} g_{1}\left(\left|x_{0}-\xi\right|\right)\left(1+|\beta| g_{1}\left(\left|x_{0}-\xi\right|\right)\right)\left|x_{0}-\xi\right|}{\left(1-L_{0}\left|x_{0}-\xi\right|\right)\left|x_{0}-\xi\right|\left(1-q\left(\left|x_{0}-\xi\right|\right)\right)}
\end{aligned}
$$

Then, by (2.9), (2.12), (2.16), (2.23), (2.25), (2.28) (2.31) and (2.35), we obtain that

$$
\begin{aligned}
\left|C_{0}\right| & \leq \frac{\alpha M_{0} M^{4}\left(\left|x_{0}-\xi\right|^{2}+|\beta-1|\left|x_{0}-\xi\right|\left|y_{0}-\xi\right|+|\beta|\left|y_{0}-\xi\right|^{2}\right)^{2}}{\left(1-L_{0}\left|x_{0}-\xi\right|\right)^{2}\left|x_{0}-\xi\right|^{3}\left(1-q\left(\left|x_{0}-\xi\right|\right)\right)^{2}} \\
& \leq \frac{\alpha \gamma M^{3} g_{1}\left(\left|x_{0}-\xi\right|\right)\left(1+|\beta| g_{1}\left(\left|x_{0}-\xi\right|\right)\right)\left|x_{0}-\xi\right|}{\left(1-L_{0}\left|x_{0}-\xi\right|\right)^{2}\left(1-\frac{L_{0}\left|x_{0}-\xi\right|}{2}\right)\left|x_{0}-\xi\right|\left(1-q\left(\left|x_{0}-\xi\right|\right)\right)^{2}}
\end{aligned}
$$

Then, using the last substep of method (1.2) for $n=0,(2.5),(2.32)$ and (2.37)-(2.40), we get in turn that

$$
\begin{aligned}
\left|x_{1}-\xi\right| & \leq\left|t_{0}-\xi\right|+|\delta|\left(\left|A_{0}\right|+\left|B_{0}\right|+\left|C_{0}\right|+\left|D_{0}\right|\right) \\
& \leq g_{3}\left(\left|x_{0}-\xi\right|\right)\left|x_{0}-\xi\right|<\left|x_{0}-\xi\right| r
\end{aligned}
$$

which shows $(2.21)$ and $x_{1} \in U(\xi, r)$. By simply replacing $x_{0}, y_{0}, t_{0}, x_{1}$ by $x_{n}, y_{n}, t_{n}, x_{n+1}$ in the preceding estimates, we arrive at estimate (2.19)-(2.21). Using the estimate $\left|x_{n+1}-\xi\right| \leq c\left|x_{n}-\xi\right|<r, c \in g_{3}\left(\left|x_{0}-\xi\right|\right) \in[0,1)$, we deduce that $x_{n+1} \in U(\xi, r)$ and $\lim _{n \rightarrow \infty} x_{n}=\xi$. Finally, to show the uniqueness part, let $Q=\int_{0}^{1} f^{\prime}\left(\xi_{1}+\theta\left(\xi-\xi_{1}\right) d \theta\right.$ for some $\xi_{1} \in D_{1}$ with $f\left(\xi_{1}\right)=0$. Using (2.8), we get in turn that

$$
\left|f^{\prime}(\xi)^{-1}\left(Q-f^{\prime}(\xi)\right)\right| \leq \frac{L_{0}}{2}\left|\xi_{1}-\xi\right| \leq \frac{L_{0}}{2} r<1
$$

Hence, $Q \neq 0$. Then, from identity $0=F(\xi)-F\left(\xi_{1}\right)=Q\left(\xi-\xi_{1}\right)$, we conclude that $\xi=\xi_{1}$. 
Remark 2.1. 1. It follows from (2.13) that condition (2.15) can be dropped, if we set

$$
M(t)=1+L_{0} t
$$

or

$$
M(t)=M=2, \text { since } t \in\left[0, \frac{1}{L_{0}}\right) .
$$

2. The results obtained here can also be used for operators $f$ satisfying autonomous differential equations $[7,10]$ of the form:

$$
f^{\prime}(x)=P(f(x)),
$$

where $P$ is a continuous operator. Then, since $f^{\prime}\left(x^{*}\right)=P\left(f\left(x^{*}\right)\right)=$ $P(0)$, we can apply the results without actually knowing $x^{*}$. For example, let $f(x)=e^{x}-1$. Then, we can choose $P(x)=x+1$.

3. The radius $\overline{r_{1}}=\frac{2}{2 L_{0}+L_{1}}$ was shown by us to be the convergence radius of Newton's method [7]

$$
x_{n+1}=x_{n}-f^{\prime}\left(x_{n}\right)^{-1} f\left(x_{n}\right), \text { for each } n=0,1,2, \ldots
$$

under the conditions $(2.12)-(2.14)$ on $D$, where $L_{1}$ is the Lipschitz constant on $D$. We have that $L \leq L_{1}$ and $L_{0} \leq L_{1}$, so $\overline{r_{1}} \leq r_{1}$. It follows that the convergence radius $r$ of the method (1.2) cannot be larger than the convergence radius $r_{1}$ of the second order Newton's method (2.43). As already noted in [7], $\overline{r_{1}}$ is at least as large as the convergence ball given by Rheinboldt [22]

$$
r_{R}=\frac{2}{3 L_{1}}
$$

In particular, for $L_{0}<L_{1}$, we have that

$$
r_{R}<\overline{r_{1}}
$$

and

$$
\frac{r_{R}}{\overline{r_{1}}} \rightarrow \frac{1}{3} \text { as } \frac{L_{0}}{L_{1}} \rightarrow 0 .
$$

That is our convergence ball $\overline{r_{1}}$ is at most three times larger than Rheinboldt's. The same value of $r_{R}$ was given by Traub [24]. 
4. It is worth noticing that method (1.2) is not changing when we use the conditions of Theorem 2.1 instead of stronger conditions used in [18]. Moreover, we can compute the computational order of convergence (COC) defined by

$$
\xi^{*}=\ln \left(\frac{\left|x_{n+1}-\xi\right|}{\left|x_{n}-\xi\right|}\right) / \ln \left(\frac{\left|x_{n}-\xi\right|}{\left|x_{n-1}-\xi\right|}\right)
$$

or the approximate computational order of convergence (ACOC) defined by

$$
\xi^{* *}=\ln \left(\frac{\left|x_{n+1}-x_{n}\right|}{\left|x_{n}-x_{n-1}\right|}\right) / \ln \left(\frac{\left|x_{n}-x_{n-1}\right|}{\left|x_{n-1}-x_{n-2}\right|}\right) .
$$

This way we obtain in practice the order of convergence in a way that avoids the bounds involving estimates using estimates higher than the first Fréchet derivative of operator $F$. Notice also that the computation of $\xi^{* *}$ does not require knowledge of $\xi$.

\section{$3 \quad$ Numerical examples}

We present numerical examples in this section.

Example 3.1. Let $X=Y=\mathbb{R}, D=\bar{U}(0,1)$. Define $f$ on $D$ by

$$
f(x)=e^{x}-1 .
$$

Then, $f^{\prime}(x)=e^{x}$ and $\xi=0$. We get that $L_{0}=e-1<L=e^{\frac{1}{L_{0}}}, L_{1}=e$, $M_{0}=M=e$ and $\gamma=1$. Then, for method (1.2) the parameters are:

$\overline{r_{1}}=0.324947, r_{1}=0.382692, r_{2}=0.07720896, r_{3}=0.0411985456, r_{q}=0.29821099$, $r=0.0411985456$

Example 3.2. Let $D=(-\infty,+\infty)$. Define function $f$ on $D$ by

$$
f(x)=\sin x .
$$

Then, we have for $\xi=0$ that $L_{0}=L=L_{1}=M_{0}=M=1$ and $\gamma=1$. Then, for method (1.2) the parameters are:

$\overline{r_{1}}=r_{1}=0.666667, r_{2}=0.336248, r_{3}=0.331336, r_{q}=0.719224, r=0.331336$. 
Example 3.3. Returning back to the motivational example at the introduction of this paper, we have that $L=L_{0}=L_{1}=146.6629073$ and $M_{0}=M=2$ and $\gamma=\frac{1}{3}$. Then, for method (1.2) the parameters are:

$$
\overline{r_{1}}=r_{1}=0.00454557, r_{2}=0.00130815, r_{3}=0.00112564, r_{q}=0.0039941 \text {, }
$$

$r=0.00112564$.

Example 3.4. Let $X=Y=\mathbb{R}$ and define function $f$ on $D=\mathbb{R}$ by

$$
f(x)=\bar{\beta} x-\bar{\gamma} \sin (x)-\bar{\delta},
$$

where $\bar{\beta}, \bar{\gamma}, \bar{\delta}$ are given real numbers. Suppose that there exists a solution $\xi$ of $f(x)=0$ with $f^{\prime}(\xi) \neq 0$. Then, we have

$$
L_{1}=L_{0}=L=\frac{|\bar{\gamma}|}{|\bar{\beta}-\bar{\gamma} \cos \xi|}, \quad M_{0}=M=\frac{|\bar{\gamma}|+|\bar{\beta}|}{|\bar{\beta}-\bar{\gamma} \cos \xi|} .
$$

Then one can find the convergence radii for different values of $\bar{\beta}, \bar{\gamma}$ and $\bar{\delta}$. As a specific example, let us consider Kepler's equation (3.1) with $\bar{\beta}=1$, $0 \leq \bar{\gamma}<1$ and $0 \leq \bar{\delta} \leq \pi$. A numerical study was presented in [13] for different values of $\bar{\gamma}$ and $\bar{\delta}$.

Let us take $\bar{\gamma}=0.9$ and $\bar{\delta}=0.1$. Then the solution is given by $\xi=$ 0.6308435 . Hence, for method (1.2) the parameters are:

$$
\begin{aligned}
& \quad \overline{r_{1}}=r_{1}=0.202387, r_{2}=0.0103345, r_{3}=0.00588456, r_{q}=0.0986948, \\
r= & 0.00588456
\end{aligned}
$$

\section{References}

[1] S. Abbasbandy, P. Bakhtiari, A. Cordero, J.R. Torregrosa, and T. Lotfi, New efficient methods for solving nonlinear systems of equations with arbitrary even order, Appl. Math. Comput., (to appear.)

[2] G. Adomian, Solving Frontier problem of physics: The decomposition method, Kluwer Academic Publishers, Dordrechet, 1994.

[3] S. Amat, S. Busquier, and J.M. Guttiérrez, Geometric constructions of iterative functions to solve nonlinear equations, J. Comput. Appl. Math., 157, (2003), 197-205.

[4] S. Amat, S. Busquier, and S. Plaza, Dynamics of the King's and Jarratt iterations, Aequationes. Math., 69, (2005), 212-213.

[5] S. Amat, M.A. Hernández, and N. Romero, A modified Chebyshev's iterative method with at least sixth order of convergence, Appl. Math. Comput., 206, (2008), 164-174. 
Vol. LIV (2016) On the local convergence ...

[6] I.K. Argyros, Improved generalized differentiability conditions for Newton-like methods, J. Complexity, 26, (2010), 316-333.

[7] I.K. Argyros, Convergence and Applications of Newton-type Iterations, Springer, 2008

[8] I.K. Argyros, Quadratic equations annd applications to Chandershekhar's and related equations, Bull Austra. Math. Soc., 32, (1985), 275-292.

[9] I.K. Argyros, Chen D., and Q. Quian, The Jarratt method in Banach space setting, J. Comput. Appl. Math., 51, (1994), 103-106.

[10] I.K. Argyros and Hilout S., Computational Methods in Nonlinear Analysis, World Scientific Publ. Comp., New Jersey, 2013

[11] A. Cordero and J.R. Torregrosa, Variants of Newton's method using fifth-order quadrature formulas, Appl. Math. Comput., 190, (2007), 686-698.

[12] A. Cordero, J.R. Torregrosa, and M.P. Vassileva, Increasing the order of convergence of iterative schemes for solving nonlinear systems, J. Comput. Appl. Math., 252, (2012), 86-94.

[13] J. M. A. Danby and T. M. Burkardt, The solution of Kepler's equation, I. Celest. Mech., 31, (1983), 95-107.

[14] J.A. Ezquerro and M.A. Hernández, New iterations of R-order four with reduced computational cost, BIT Numer Math., 49, (2009), 325-34.

[15] J.M. Gutiérrez and M.A. Hernández, Comput. Math. Appl., 36, (1998), 1-8.

[16] E. Hansen and M. Patrick, A family of root finding methods, Numer. Math., 27, (1977), 257-269.

[17] P. Jarratt, Some fourth order multipoint methods for solving equations, Math. Comput., 20, (1966), 434-437.

[18] M. Kansal, V. Kanwar, and S. Bhatia, New modifications of Hansen-Patrick's family with optimal fourth and eighth orders of convergence, Appl. Math. Comput., 269, (2015), 507-519.

[19] B. Neta, M. Scott, and C. Chun, Basins of attraction for several methods to find simple roots of nonlinear equations, Appl. Math. Comput., 218, (2012), 10548-10556.

[20] M.S. Petković, B. Neta, L.D. Petković, and J. Dzunić, Multipoint Methods for Solving Nonlinear Equations, Amsterdam, 2013.

[21] F.A. Potra and V. Pták, Research Notes in Mathematics, 103, (1984.)

[22] W.C. Rheinboldt, An adaptive continuation process for solving systems of nonlinear equations, Mathematical models and numerical methods (A.N.Tikhonov et al. eds.) pub.3, 19, (1977), 129-142

[23] J.R. Sharma, R.K. Guha, and R. Sharma, An efficient fourth-order weightedNewton method for systems of nonlinear equations, Numer. Algor., 62, (2013), 307323.

[24] J.F. Traub, Iterative Methods for the Solution of Equations, Prentice-Hall, New Jersey, 1964 
Ioannis K. Argyros

Department of Mathematical Sciences,

Cameron University, Lawton, OK 73505, USA

E-mail: iargyros@cameron.edu

Munish Kansal

University Institute of Engineering and Technology,

Panjab University, Chandigarh-160-014, India

E-mail: mkmaths@gmail.com

V. Kanwar

University Institute of Engineering and Technology,

Panjab University, Chandigarh-160-014, India

E-mail: vmithil@yahoo.co.in

Received: 3.03.2016

Accepted: 21.03.2016 